\title{
A Wideband Spiral UHF Coupler with Tuning Nodules for Partial Discharge Detection
}

DOI:

10.1109/TPWRD.2018.2883828

\section{Document Version}

Accepted author manuscript

Link to publication record in Manchester Research Explorer

\section{Citation for published version (APA):}

Zachariades, C., Shuttleworth, R., Giussani, R., \& Loh, T-H. (2018). A Wideband Spiral UHF Coupler with Tuning Nodules for Partial Discharge Detection. IEEE Transactions on Power Delivery.

https://doi.org/10.1109/TPWRD.2018.2883828

\section{Published in:}

IEEE Transactions on Power Delivery

\section{Citing this paper}

Please note that where the full-text provided on Manchester Research Explorer is the Author Accepted Manuscript or Proof version this may differ from the final Published version. If citing, it is advised that you check and use the publisher's definitive version.

\section{General rights}

Copyright and moral rights for the publications made accessible in the Research Explorer are retained by the authors and/or other copyright owners and it is a condition of accessing publications that users recognise and abide by the legal requirements associated with these rights.

\section{Takedown policy}

If you believe that this document breaches copyright please refer to the University of Manchester's Takedown Procedures [http://man.ac.uk/04Y6Bo] or contact uml.scholarlycommunications@manchester.ac.uk providing relevant details, so we can investigate your claim.

\section{OPEN ACCESS}




\title{
A Wideband Spiral UHF Coupler with Tuning Nodules for Partial Discharge Detection
}

\author{
Christos Zachariades, Roger Shuttleworth, Member, IEEE, Riccardo Giussani, Member, IEEE, \\ Tian-Hong Loh, Senior Member, IEEE
}

\begin{abstract}
This paper reports on the development and testing of a wideband spiral-shaped coupler for Partial Discharge (PD) detection in the UHF spectrum. The coupler design, which is based on the log-periodic spiral slot antenna, has been optimized using finite element simulation. The following parameters have been optimized: feed location, arm termination shape, width of arm root and termination. The unique feature of the coupler is the inclusion of tuning nodules on the spiral arms, which widen its bandwidth without the need for additional components. Tests in an anechoic chamber have shown close agreement with the simulation results. The coupler exhibits a wide bandwidth (S11 < - 9.55 dB) between $0.5-2.6 \mathrm{GHz}$ and a peak gain of $4.8 \mathrm{dBi}$ (at $1.35 \mathrm{GHz}$ ), while using an infinite balun coaxial feed which allows for an input impedance of $50 \Omega$. PD testing in the laboratory has shown that the phase resolved PD patterns acquired with the coupler are very similar to those captured with conventional PD sensors. An application of the coupler as an internal sensor in a gas-insulated busduct is detailed and a bandwidth comparison with other UHD PD sensors is provided.
\end{abstract}

Index Terms - condition monitoring, finite element analysis (FEA), gas-insulated switchgear (GIS), partial discharge (PD), sensor, spiral antenna, tuning nodules, UHF couplers.

\section{INTRODUCTION}

$\mathrm{T}$ he reliability of modern high voltage equipment is continuously improving due to advances in design and manufacturing technologies. Nevertheless, the possibility of failure due to Partial Discharge (PD) can never be completely eliminated. Furthermore PD failure risks increase as the voltage level increases and as equipment ages. Most high voltage equipment currently in service is not new since such equipment was designed to last in excess of forty years in many occasions. To mitigate against the risk of equipment failure, PD monitoring and diagnostics are widely employed to determine the condition of the electrical insulation of critical assets such as rotating machines, switchgear, transformers and cables [1].

PD measurements can be conducted according to IEC 60270 [2] using a variety of sensors such as High Voltage Coupling Capacitors (HVCC), Rogowski Coils, Transient Earth Voltage detectors, and High Frequency Current Transformers (HFCT)

Manuscript received August 05, 2018; revised October 12, 2018; accepted November 17, 2018. This work was co-funded by the UK's Innovation Agency, Innovate UK. Paper no. TPWRD-00915-2018.

C. Zachariades is with the University of Manchester (e-mail: christos.zachariades@manchester.ac.uk), Manchester, M13 9PL, UK.

R. Shuttleworth is with the University of Manchester (e-mail: roger.shuttleworth@manchester.ac.uk), Manchester, M13 9PL, UK.

R. Giussani is with High Voltage Partial Discharge Ltd (e-mail: riccardo.giussani@hvpd.co.uk), Salford, M502UW, UK.

T.-H. Loh is with the UK National Physical Laboratory (e-mail: tian.loh@npl.co.uk), Teddington, Middlesex, TW11 0LW, UK.

Color versions of one or more of the figures in this paper are available online at http://ieeexplore.ieee.org. Digital Object Identifier .
[3]. However, the installation of such sensors is not suitable for certain equipment such as Gas-Insulated Switchgear (GIS) and power transformers, since their construction tends to reduce the sensitivity of the measurement system. The components of interest are enclosed within sealed metallic enclosures which are either pressurized or filled with oil, making the use of conventional sensors impractical if not impossible. For these assets the UHF method for PD detection has been used extensively over the past few decades with very positive results $[4,5]$. Sensitivity comparable to conventional methods is achievable [2] since interference from external noise sources has minimal effect on PD signals which are 'trapped' within the metallic enclosures of the equipment. Nevertheless, UHF PD detection can be very challenging due to a multitude of reasons that can affect the detected frequency spectrum of the PD signals which can consequently lead to misclassification of defects. These include the type and dimensions of the defects, their location within the equipment as well as the properties of the couplers and measurement instruments [6].

A variety of UHF sensors are available for PD detection in HV apparatus such as the standard-type and non-conventional sensors used for GIS [7] or the UHF probes used for power transformers [8]. However, many of these were not originally designed for PD detection and have been adapted from other applications, for example the standard-type disk sensor which was initially designed to measure fast transients in GIS.

The paper describes the development and testing of a Wideband Spiral UHF Coupler which can be used for PD detection in the UHF spectrum. The coupler provides improved characteristics compared to other UHF PD sensors [8] while being simple and cheap to manufacture. With its low return loss over most of the UHF spectrum it does not require additional components for impedance matching with PD monitoring instruments and can provide an additional option for more effective PD diagnosis. The coupler design has been optimized using finite element simulation and incorporates a unique feature, the tuning nodules. These allow its bandwidth to be extended without the need for additional components or impedance matching feeding structures that would add to its thickness. The results of testing in both an anechoic chamber and a HV laboratory are presented as well as a comparison of its bandwidth with that of other UHF PD sensors.

\section{COUPLER Design}

\section{A. Dimensions}

The spiral coupler dimensions were constrained primarily by its intended applications, e.g. as an internal sensor for PD 
diagnosis in GIS and power transformers. Since the coupler is to be installed within high voltage equipment in order to detect $\mathrm{PD}$, a small thickness is essential to avoid infringing the electrical clearances. Furthermore, to ensure compatibility with a variety of HV equipment its length and width had to be maintained as small as possible while at the same time ensuring wideband characteristics within the UHF spectrum. Additional considerations included the cost and ease of manufacture. Therefore, it was decided to construct the coupler on a printed circuit board (PCB) with the following dimensions:

- Length: $200 \mathrm{~mm}$

- Width: $200 \mathrm{~mm}$

- Thickness (of substrate): $1.6 \mathrm{~mm}$

The design of the coupler is based on the logarithmic or equiangular spiral slot antenna. The length, width and pitch of each arm of the spiral are defined by the following set of parametric equations [9]:

$$
\begin{array}{ll}
x(t)=1.175^{t} \cos (t) & y(t)=1.175^{t} \sin (t) \\
x(t)=1.185^{t} \cos (t) & y(t)=1.185^{t} \sin (t)
\end{array}
$$

with equations (1) and equations (2) defining the inner and outer edges of the slotted spiral arms respectively.

Spiral slot antennas built with one and a half to three turns have been shown to exhibit good radiation characteristics [10]. Therefore, it was decided to fix the number of turns for the spiral coupler to two to minimize the number of variables that would require optimization.

\section{B. Coupler Feed}

In addition to the overall dimensions, an equally important design element for the coupler is the feed structure. The overwhelming majority of equipment used for PD detection (e.g. oscilloscopes, frequency downconverters, and other sensors) are $50 \Omega$ terminated and therefore that was a requirement for the coupler feed to ensure compatibility.

However, theoretical calculations have shown that the input impedance spiral antennas is approximately $188 \Omega$ [11] (from Babinet's Principle [12]) while experimental measurements have shown this to be close to $164 \Omega$ [10]. In many occasions to achieve impedance matching a coaxial balun [13], a tapered microstrip transmission line [14] or a coplanar waveguide [15] can be used to feed the antenna from the middle of the spiral. Such feed structures would add to the thickness of the coupler which, as mentioned earlier, needed to be kept as small as

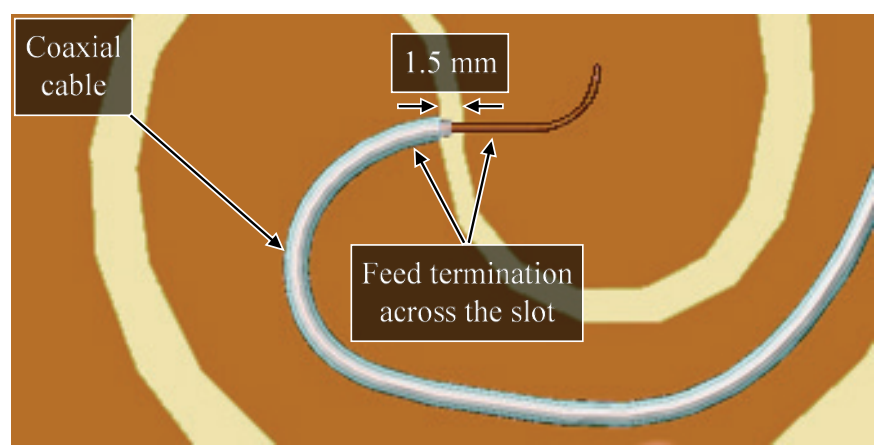

Fig. 1. Spiral UHF coupler feed using an infinite balun constructed from RD316 coaxial cable. possible.

A different feed method was therefore chosen, the infinite balun $[11,16]$. It was constructed from a balanced transmission line made from RD316 coaxial cable embedded in the ground plane and feeding the slot at the center of the spiral (Fig. 1). The cable was terminated with a crimped SMA $50 \Omega$ connector which was soldered onto the PCB outer edge to provide structural rigidity. With the infinite balun the shield of the coaxial cable essentially becomes part of the spiral antenna and the balun does not allow currents excited outside the cable to reach the input [11]. It allows use of the full extent of the coupler's bandwidth [16].

\section{OPTIMIZATION WITH SIMULATION}

Following the definition of its main geometric parameters a three-dimensional model of the coupler was constructed using a Computer Aided Design (CAD) software package. The model incorporated high fidelity details such as the thickness of the PCB substrate, the coaxial cable for the feed and the SMA connector. The model was imported into COMSOL Multiphysics to perform optimization of the various parameters of the antenna in the virtual domain before constructing a physical prototype. The computation was performed between $0.2 \mathrm{GHz}$ and $3.3 \mathrm{GHz}$ in steps of $0.1 \mathrm{GHz}$.

The aim was to achieve a wide coupler bandwidth while maintaining a low reflection coefficient that would eliminate the requirement for additional impedance matching measures to be implemented. Hence the bandwidth of the coupler was defined based on the requirement for the S11 parameter to be lower than $-9.55 \mathrm{~dB}$ which corresponds to a Voltage Standing Wave Ratio (VSWR) of less than 2.0.

\section{A. Simulation Setup}

The simulations described in the following sections were performed with the RF Module available in COMSOL Multiphysics. This module formulates and solves the differential form of Maxwell's equations using the Finite Element Method (FEM) taking into account the initial and boundary conditions. Specifically, the Electromagnetic Waves, Frequency Domain interface was used that solves the timeharmonic wave equation for the electric field:

$$
\nabla \times\left(\mu_{r}^{-1} \nabla \times \mathbf{E}\right)-k_{0}{ }^{2} \varepsilon_{r} \mathbf{E}=0
$$

where $\mathbf{E}$ is electric field, $\varepsilon_{r}$ is the relative permittivity, $\mu_{r}$ is the relative permeability and $k_{0}$ is the wave number of free space.

All metallic surfaces such as the metallization on the PCB, the conductor and shield of the coaxial cable, and the metallic parts of the SMA connector were modelled as lossless metallic surfaces using the Perfect Electric Conductor (PEC) boundary condition which sets the tangential component of the electric field to zero:

$$
\hat{\mathbf{n}} \times \mathbf{E}=0
$$

Excitation for the model was achieved with the use of the Lumped Port (Coaxial) boundary condition, specifying the location at which energy enters and exits the model at the SMA 


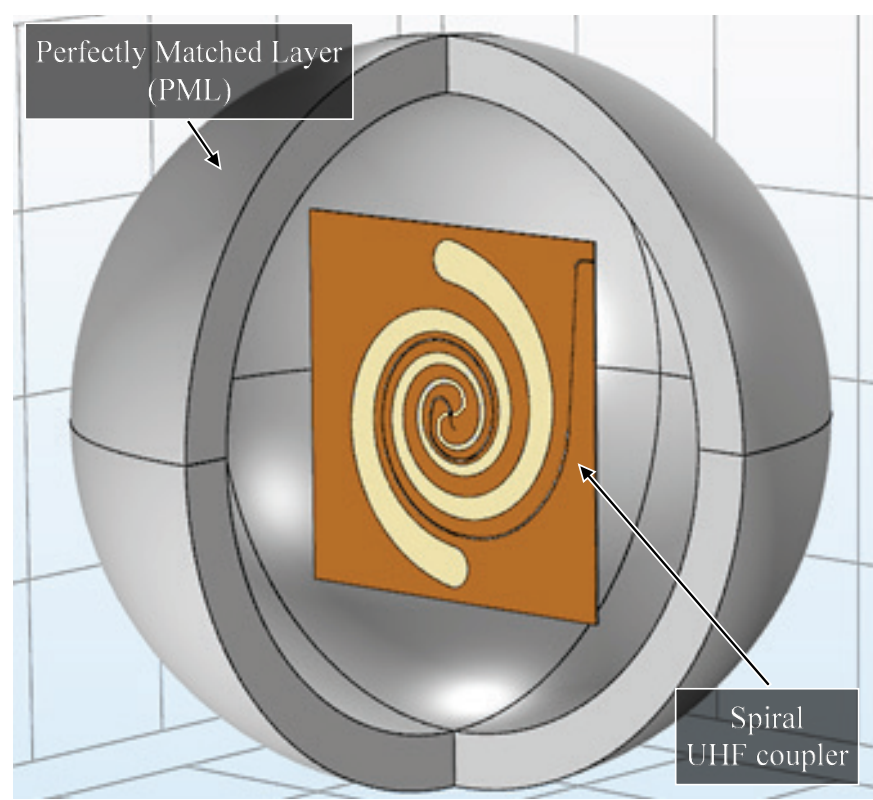

Fig. 2. Computation domain including the spiral UHF coupler geometry. The outer PML domain absorbs all outgoing wave energy without impedance mismatch while the model is excited by a $50 \Omega$ coaxial lumped port.

connector. This essentially sets the coaxial feed cable to operate in the Transverse Electro-Magnetic (TEM) mode. The characteristic impedance of the port was set to $50 \Omega$ which matches that of the connector.

The coupler model was placed within a spherical domain which itself was surrounded by a Perfectly Matched Layer (PML) domain. By applying a complex coordinate scaling to a layer of virtual domains that surround the simulation domain, the PML absorbs all outgoing wave energy without impedance mismatch. This avoids energy being reflected back from the boundary, effectively allowing the simulation to be performed as if the antenna is hanging in free space. The computation domain can be seen in Fig. 2.

The properties of the insulating materials required for the computation are the electrical conductivity, relative permittivity and relative permeability. These properties, which do not vary significantly over the frequency range covered by the simulation, are shown in Table I.

The default elements used by COMSOL to discretize the geometry are second-order Lagrangian elements. An empirical rule of thumb is to use five such elements per wavelength [1719]. While it is possible to parametrize the mesh size based on frequency to reduce memory requirements this feature was not utilized in order to ensure the quality of the mesh. Manual meshing was performed using a combination of mesh types, such as triangular, tetrahedral and swept, to account for the large size differences between model parts (e.g. the long,

TABLE I

MATERIAL PROPERTIES FOR COMPUTATION

\begin{tabular}{lcccc}
\hline \hline Component & Material & $\begin{array}{c}\text { Conductivity } \\
(\mathrm{S} / \mathrm{m})\end{array}$ & $\begin{array}{c}\text { Relative } \\
\text { permittivity }\end{array}$ & $\begin{array}{c}\text { Relative } \\
\text { permeability }\end{array}$ \\
\hline $\begin{array}{l}\text { PCB substrate } \\
\begin{array}{l}\text { Balun } \\
\text { insulation }\end{array}\end{array}$ & FR4 & 0.004 & 4.5 & 1.0 \\
$\begin{array}{l}\text { Enclosing } \\
\text { domain }\end{array}$ & Air & 0 & 2.3 & 1.0 \\
\hline \hline
\end{tabular}

small-diameter feed cable, and the flat, thin PCB). The mesh size was determined by the highest frequency $(3.3 \mathrm{GHz})$ to ensure the mesh was appropriate for the shortest wavelength. Additionally it was made sure that at least two second-order elements were used per $90^{\circ}$ arc [20]. The main mesh parameters were the following:

- Number of elements: 9.31 million

- Minimum element size: $0.06 \mathrm{~mm}$

- Maximum element size: $14 \mathrm{~mm}$

\section{B. Optimization of Feed Location}

The fact that the coupler is not fed from the underside of the PCB opens up different possibilities for the location of the feed starting position around the periphery of the ground plane. To identify the optimal location, variations of the coupler model with different feed positions were simulated as seen in Fig. 4. The feed positions considered (in relation to Fig. 2) were the following:

(a) At the top right corner of the square PCB.

(b) At the middle of the right hand side of the PCB.

(c) At the top and middle of the PCB near the termination of the slotted spiral arm.

The results presented in Fig. 3 show how the feed location affects the frequency response of the spiral coupler. The Right feed location allows for a reflection coefficient below $-10 \mathrm{~dB}$ from $0.6 \mathrm{GHz}$ but limits the bandwidth of the antenna with the S11 parameter rising above $-10 \mathrm{~dB}$ after $2.0 \mathrm{GHz}$. On the other hand, the Top feed location allows the antenna to have a better response characteristic at the top-end of the frequency range but a poorer characteristic at the bottom-end of the spectrum. The Corner feed location provides the best frequency response across the entire frequency range of interest, with the reflection coefficient lower than $-10 \mathrm{~dB}$ from $0.6 \mathrm{GHz}$ to $2.3 \mathrm{GHz}$.

\section{Optimization of Arm Termination}

Another geometric aspect of the spiral coupler that required

(a) Corner feed
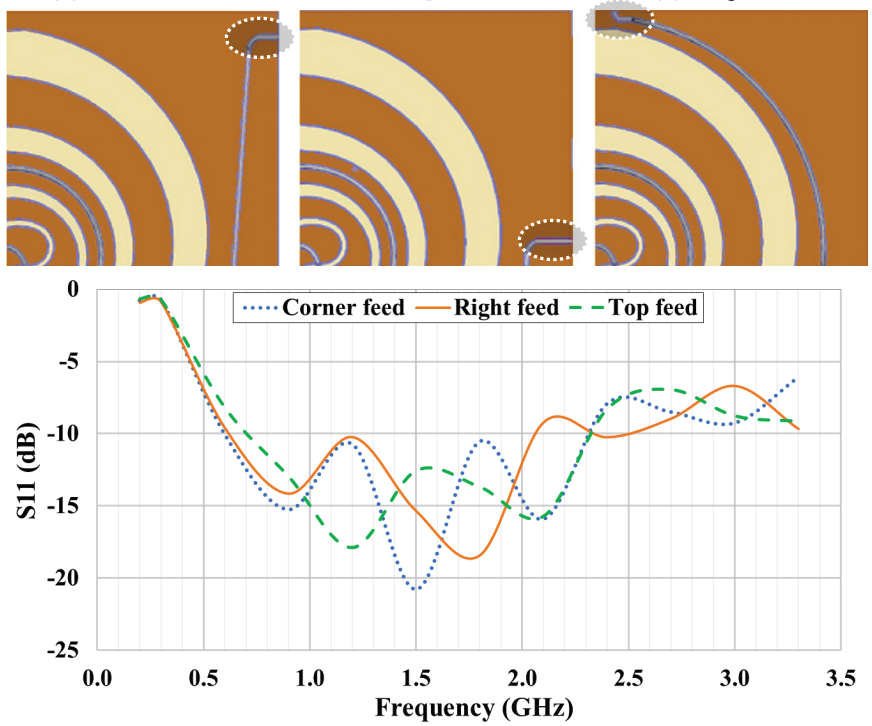

Fig. 3. Variations of the feed location and their effect on the reflection coefficient of the spiral UHF coupler. 
optimization was the shape of the spiral arm termination. It has been documented [10] that by tapering the ends of the spiral arms a better matching termination is achieved. To investigate how the arm termination affects the frequency response four coupler models were simulated, each incorporating a different arm termination shape: Unterminated, Flared (or tapered), Convex and Concave, as seen in Fig. 4.

The simulation results show that, with the exception of the flared termination, the difference in the frequency response of the coupler due to the difference in arm termination shape is very subtle. The flared termination resulted in worse performance at the low end of the spectrum and therefore it was not deemed suitable for the spiral coupler. Out of the other three termination shapes the Convex was chosen due to its marginally better performance in the $0.5 \mathrm{GHz}-0.7 \mathrm{GHz}$ range.

\section{Optimization of Arm Width}

While the length of the arms of the spiral coupler is restricted by its overall dimensions (defined in Section II.A above) their width can vary, providing the infinite balun feed can be accommodated. The slotted arms, as shown in Fig. 2, start narrow at the center of the spiral and become progressively wider until they terminate near the edge of the board. Simulations with varying the arm widths at both the root (at the center of the spiral) and the termination (Fig. 5) were carried out to investigate the effect of arm width on the frequency response of the coupler. The results can be seen in Fig. 6 .

The width of the arm at the termination affects predominantly the response at the lower end of the spectrum. Increasing the arm termination width shifts the bandwidth to lower frequencies but it also reduces its effective range. Conversely a smaller arm termination width results in a better response at higher frequencies. For the spiral coupler, an arm termination width of $19 \mathrm{~mm}$ was chosen because it resulted in the widest bandwidth.

The width of the arm at the center of the spiral starts having
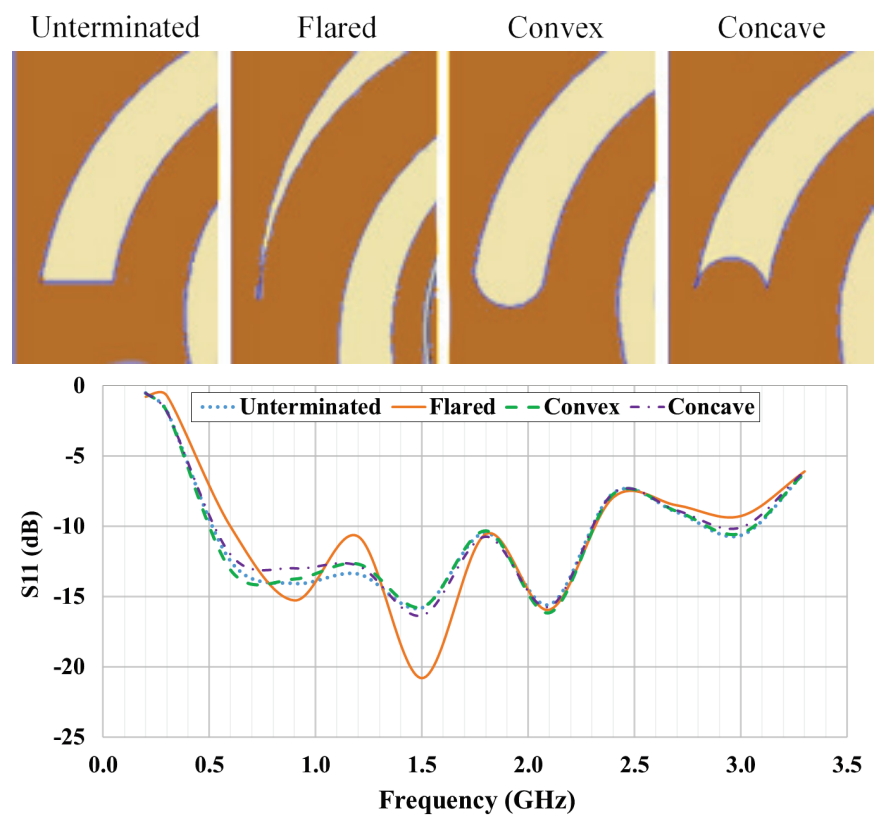

Fig. 4. Effect of the arm termination shape to the frequency response of the spiral UHF coupler. a significant effect on the frequency response above $1.2 \mathrm{GHz}$ as shown in Fig. 5. Making the arm root width smaller widens the bandwidth of the coupler since it results in a smaller reflection coefficient. Despite the improved performance of smaller arm root widths, an arm root width of $1.5 \mathrm{~mm}$ was selected for the spiral coupler. Smaller widths were deemed unsuitable for practical applications since they would be too small to accommodate the termination of the coaxial cable used for the infinite balun without using expensive manufacturing processes.

\section{E. Tuning Nodules}

After the optimization of the various parameters described earlier it became apparent that more drastic solutions would be required in order to further improve the bandwidth of the coupler. Of particular interest was the relatively high reflection coefficient appearing at certain frequencies despite the implemented optimizations, for example at $2.45 \mathrm{GHz}$. To investigate the reason behind this effect, the electric field plots at the frequencies resulting in high reflection coefficient were compared with the plots at frequencies where the reflection coefficient was lower. It was observed that at frequencies where the reflection coefficient was high (e.g. $2.45 \mathrm{GHz}$ ) the electric field exhibited a minimum value at a distance of quarter-wavelength from the arm termination (Fig. 6), indicating reduced radiating efficiency.
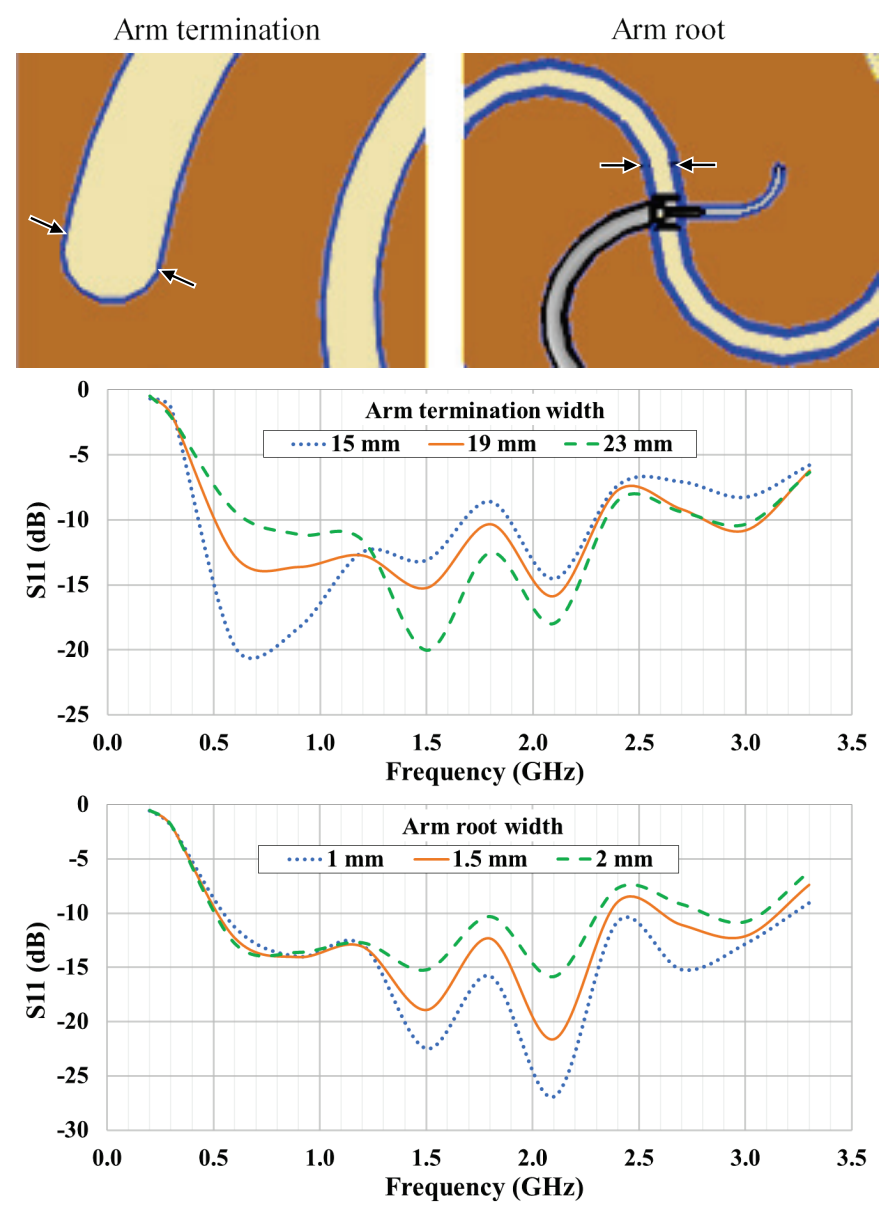

Fig. 5. Effect of the arm width (termination and root) to the frequency response of the spiral UHF coupler. 
To mitigate the aforementioned effect and improve the frequency response of the coupler, a unique feature was added to the arms of the spiral, the tuning nodules. These are semicircular cut-outs on the metallization of the PCB which effectively make the slotted spiral arms wider at specific locations along their length. The nodules are positioned with their centres located away from the arm termination at a distance of a quarter-wavelength (or multiples of it) at the frequency of interest. The number as well as the size of the nodules were optimized using simulation. The tuning nodules provide a simple way to fine-tune the frequency response of the coupler by minimising the current-wave reflections from the ends of the spiral arms as they perform a similar function to resistive loading [21]. This eliminates the need to add additional components to the coupler reducing both the cost and complexity of manufacture.

The effect of the tuning nodules on the coupler electric field distribution can be seen in Fig.6 where the local electric field minimum near the arm termination at $2.45 \mathrm{GHz}$ was eliminated. The improvement in the frequency response of the coupler due to the addition of the tuning nodules is shown in Fig. 7. The strategic placement of the tuning nodules on the arms of the spiral coupler eliminates the electric field minimum at the frequencies of interest. As a result the frequency response becomes flatter and the bandwidth is extended by approximately $0.8 \mathrm{GHz}$ at the higher end of the spectrum.

\section{TESTING AND COMPARISON WITH SIMULATION}

An advanced prototype of the coupler was constructed based on the outcome of the optimization process and can be seen in

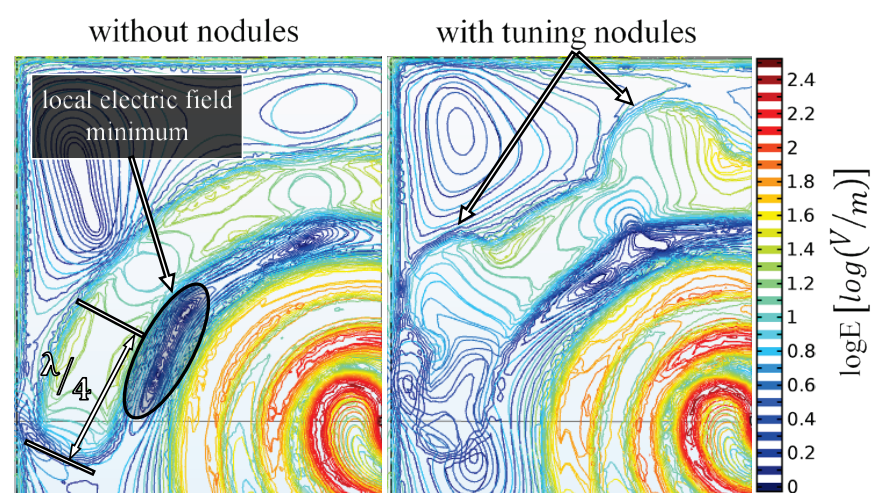

Fig. 6. Electric field contour plots showing local minimum at a distance of quarter-wavelength from the arm termination at $2.45 \mathrm{GHz}$ resulting in reduced radiating efficiency. The electric field minimum is eliminated after the addition of tuning nodules on the spiral UHF coupler arms.

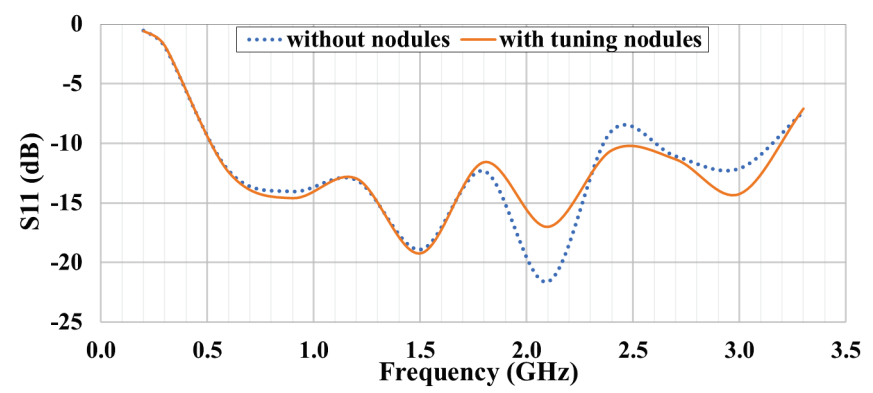

Fig. 7. Improvement in the frequency response of the spiral UHF coupler due to the addition of tuning nodules on the spiral arms. The bandwidth is effectively extended by approximately $0.8 \mathrm{GHz}$.
Fig. 8. Testing was performed in two stages. Firstly, the coupler was tested in an anechoic chamber to measure its radiation characteristics and validate the results obtained by the simulations. Secondly, it was tested in a bespoke test rig to check its PD detection capabilities.

\section{A. Measurement of Coupler Characteristics and Comparison with Simulation}

Testing of the coupler radiation performance was conducted at the National Physical Laboratory. The anechoic chamber was $7.15 \mathrm{~m}$ long, $6.25 \mathrm{~m}$ wide, and $6.25 \mathrm{~m}$ high. The following parameters were measured between $500 \mathrm{MHz}$ and $3 \mathrm{GHz}$ with $1 \mathrm{MHz}$ frequency resolution:

- S-parameters

- Gain

- 3D radiation pattern ( $5^{\circ}$ angular resolution)

- Polarization

For gain measurement, the three-antenna-method was utilized [22]. The equipment used for the test was the following:

- $\quad$ Reference (Tx) antenna: USLP 9143

- VNA: Agilent N5242A PNA-X

- Positioner: Orbit, roll-over-azimuth

- Mast: Kevlar, $130 \mathrm{~mm}$ in diameter

The coupler was mounted on the positioner at $2.51 \mathrm{~m}$ above ground. The separation between the reference and under-test antennas was measured to be $2.22 \mathrm{~m}$. The setup can be seen in Fig. 9.

The variation of coupler gain with frequency and a comparison with computed values can be seen in Fig. 10. The computed and measured values generally follow a similar trend. Their difference can be attributed to the imperfect infinite balun and the effect of the unwanted common mode on the cable [23]. Based on the measurements, the coupler exhibits a higher than $0 \mathrm{dBi}$ gain in the range of $0.5 \mathrm{GHz}-2.7 \mathrm{GHz}$, with a peak gain of $4.8 \mathrm{dBi}$ observed at $1.35 \mathrm{GHz}$.

The computed and measured radiation patterns are also in close agreement over the entire UHF spectrum and, as expected, the shape of the radiation pattern does not vary significantly

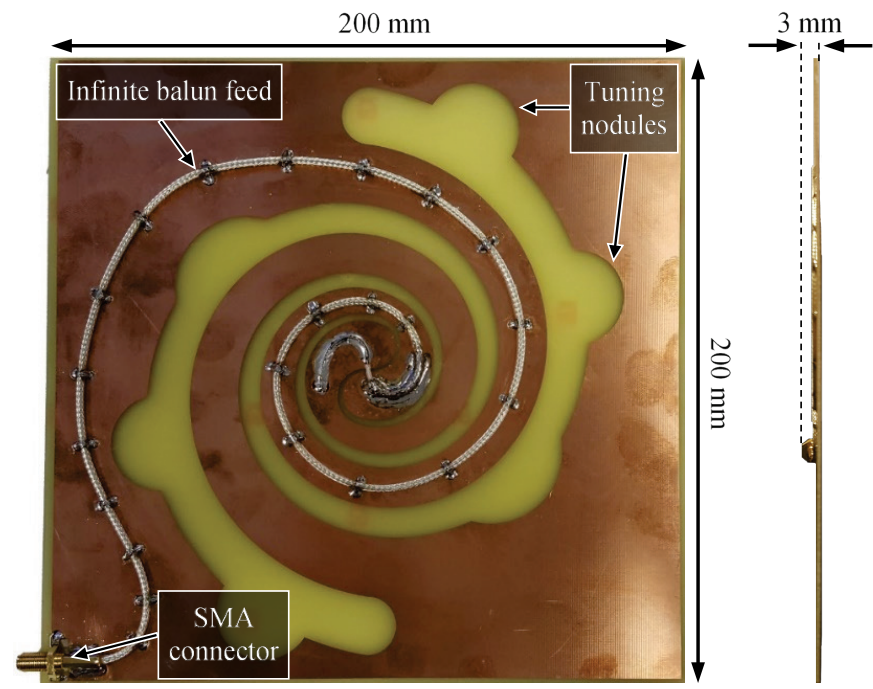

Fig. 8. Spiral UHF coupler prototype, showing main components and dimensions 


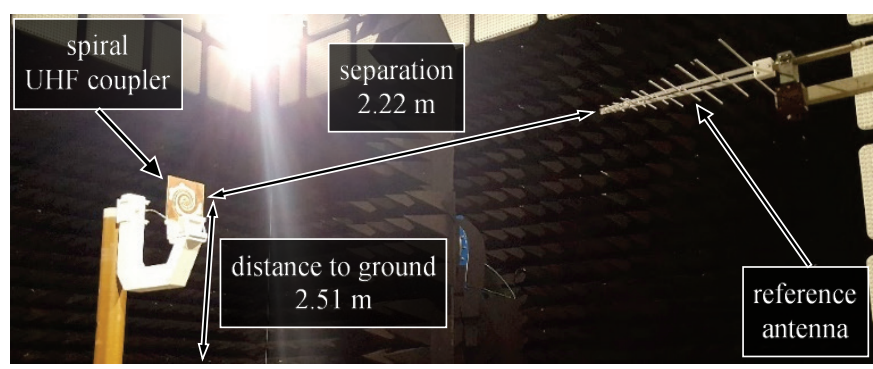

Fig. 9. Spiral UHF coupler test setup in the anechoic chamber of the National Physical Laboratory.

with frequency. The coupler exhibits a relatively wide main beam width which is $120^{\circ}$ at its minimum. Furthermore, no side lobes have been observed.

The frequency response of the coupler is shown in Fig. 11. The reflection coefficient (S11 parameter) remains below -9.55 $\mathrm{dB}$ for much of the frequency range of interest. Hence, the bandwidth of the coupler, as defined in Section III, was determined to be $0.5 \mathrm{GHz}-2.6 \mathrm{GHz}$.

\section{B. Testing of the Coupler's PD Detection Capabilities}

Since the main function of the spiral UHF coupler is PD detection in HV equipment, a bespoke test rig was constructed to test its capabilities. The rig (Fig. 12) consists of a barrel, which is similar to a short section of GIS tank, a $20 \mathrm{kV}$ transformer, and a discharge cell emulating a typical GIS defect, a metallic protrusion on the HV conductor [24] (i.e. a point-plane electrode arrangement). A $16 \mathrm{kV} \mathrm{HVCC,} \mathrm{was}$ connected to the HV terminal of the transformer while the coupler was positioned at the top of the rig. The signal outputs of both the coupler and the HVCC were connected to a $3 \mathrm{GHz}$ oscilloscope using RG223 coaxial cables of equal length. A quadrupole was used to separate the power frequency signal (sinewave) and the PD measurement signal. The testing was conducted in a fully shielded room, with background noise level smaller than $2 \mathrm{pC}$, under ambient conditions (temperature: $21^{\circ} \mathrm{C}$, relative humidity: $44 \%$ ). The transformer and HVCC were tested individually and found to be PD-free up to $12 \mathrm{kV}$. The PD inception voltage for the discharge cell was $4.3 \mathrm{kV}$.

The PD results obtained with the HVCC and the UHF coupler are shown in Fig. 13. The magnitude of the discharges recorded with the HVCC reaches approximately $200 \mathrm{mV}$, and is much higher than the PD magnitude recorded with the coupler that peaks at $8 \mathrm{mV}$. The difference is to be expected

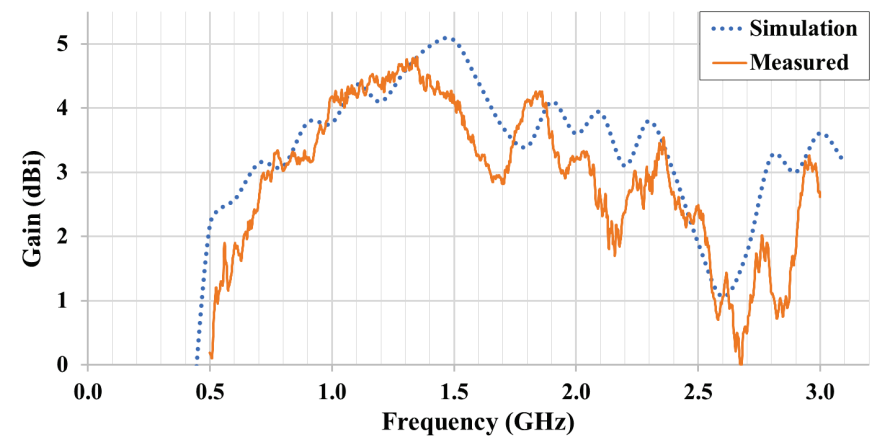

Fig. 10. Spiral UHF coupler gain over the $0.5-3 \mathrm{GHz}$ frequency range showing good match between the computed and measured results.

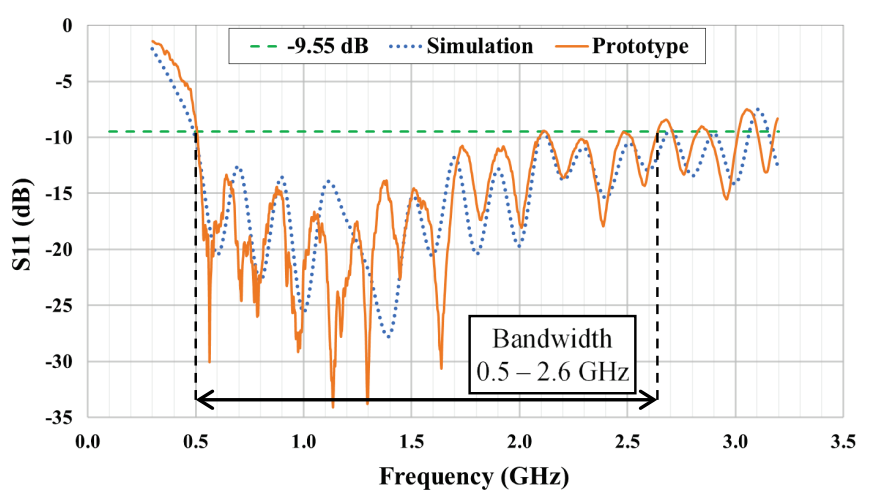

Fig. 11. Comparison between the computed and measured coupler S11 over the $0.5-3 \mathrm{GHz}$ frequency range. The spiral coupler operates at $0.5-2.6 \mathrm{GHz}$ with a $\mathrm{S} 11<-9.55 \mathrm{~dB}$.

since the HVCC is galvanically connected to the source of the discharges while the coupler only detects the energy radiated from the discharge source. Despite the very different detection approach between the two sensors, the phase resolved PD pattern from both is very similar, indicating that the coupler works as intended.

\section{APPlication to POWER System APPARATUS}

\section{A. Application as Internal PD Sensor in GIS}

The main intended use of the spiral UHF sensor is as an internal PD sensor in GIS and power transformers. The modular construction of the majority of HV GIS however allows for increased flexibility when it comes to the installation of the sensor when compared to transformers. For GIS, the sensor can be located at any point within a bay or gas-insulated busduct (GIB). It can be installed behind the covers of access openings or behind the end plates used to seal the modular sections (Fig. 14). The signal cable can then be routed through a gastight bushing plate to a terminal box, both of which are available from GIS manufacturers for use with instrumentation transformers. The sensor can be retrofitted on GIS without existing PD detection capability but it will require an outage and for the equipment to be degassed.

Following the installation, the sensitivity of the coupler

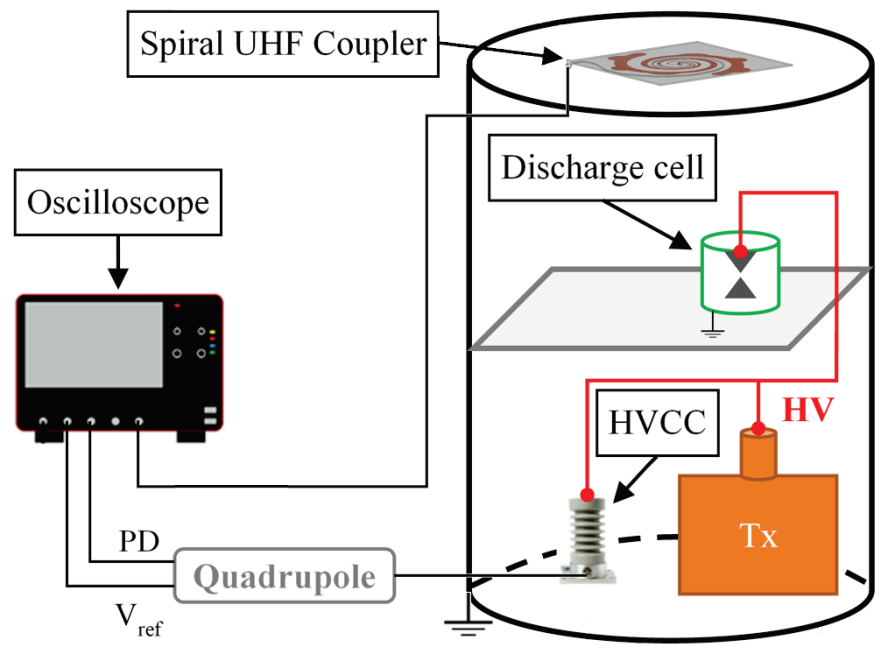

Fig. 12. Experimental setup used to test the PD detection capabilities of the spiral UHF coupler. It closely resembles a typical PD test for GIS. 

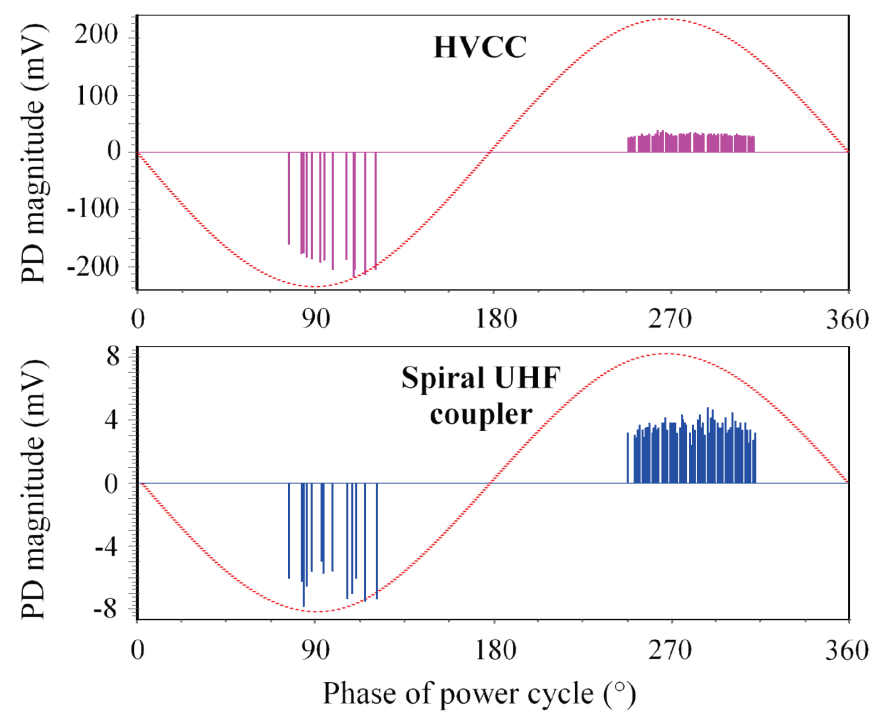

Fig. 13. Similar phase resolved PD patterns of the well-known HVCC sensor and the spiral UHF coupler (after denoising), underlining the latter's PD detection capability.

needs to be verified following the procedure outlined in [25], since calibration of UHF PD detection systems to apparent charge in $\mathrm{pC}$ is not possible. The signal output of the spiral coupler can be connected either directly to wide-band measurement instruments, such as high-frequency oscilloscopes, or to narrow-band instruments via a UHF downconverter. A PD pulse detected by the spiral coupler installed in a GIB connected to a $3 \mathrm{GHz}$ oscilloscope, is shown in Fig. 15. The figure also shows the reduced sensitivity of the UHF PD detection method to noise, which is one of its main benefits.

\section{B. Comparison with Existing UHF PD Sensors}

To further examine the capabilities of the spiral UHF coupler its bandwidth was compared with that of other UHF PD sensors that are commonly used for PD detection in GIS. These sensors, shown in Fig. 16, include both internal/permanent (disk) as well as external/portable (barrier, monopole) sensors [7]. The spiral coupler outperforms all other sensors in terms of bandwidth by a substantial margin. Therefore it has a greater chance of detecting a variety of defects without the need to use multiple sensors. Additionally, the wide bandwidth allows for the implementation of simple noise rejection techniques such as hardware filtering. Compared to the other internal sensor, the disk coupler, the spiral coupler is lighter, cheaper and easier to manufacture. It can be printed on a PCB using standard

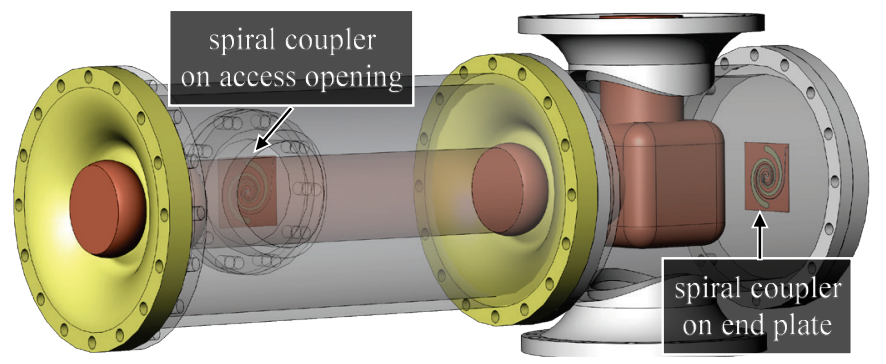

Fig. 14. Installation of the UHF spiral coupler as an internal sensor within a $420 \mathrm{kV}$ modular busduct section. The coupler can be installed behind the covers of access openings or behind the end plates of modular sections.

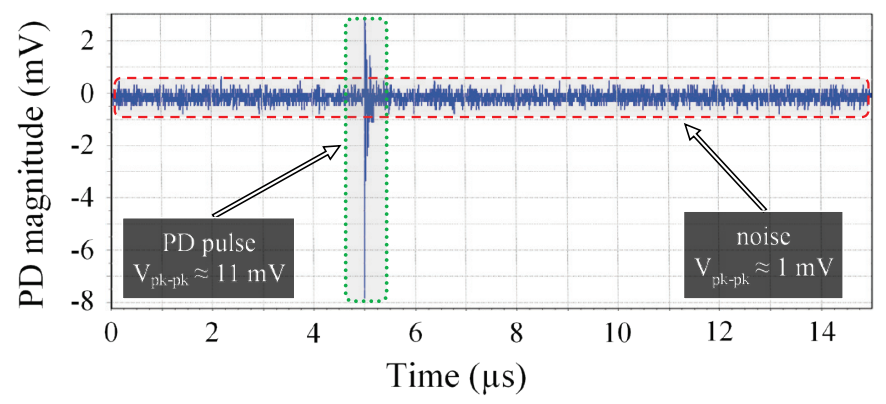

Fig. 15. PD pulse (rise time $\approx 0.3 \mathrm{~ns}$ ) due to metallic protrusion on the HV conductor, detected by the spiral UHF coupler within a gas-insulated busduct.

equipment and, unlike the disk, it does not require machining. Due to its construction, it is very thin $(3 \mathrm{~mm})$ making it ideal for installation in HV GIS, which are increasingly becoming more compact, without compromising electrical clearances.

It is important however to note that not all the sensors of Fig. 16 can be used under the same circumstances. Contrary to the Barrier and Monopole UHF sensors, the spiral UHF coupler cannot be retrofitted or installed on live equipment. Also the larger dimensions of the spiral coupler compared to the disk sensor prohibit its installation on medium and low voltage equipment that are physically smaller.

\section{CONCLUSION}

A wideband spiral UHF coupler has been developed for partial discharge detection in the UHF spectrum. Its main specifications are the following:

- Dimensions (L x W x H): $200 \times 200 \times 3$ mm

- Bandwidth (VSWR < 2): $0.5-2.6 \mathrm{GHz}$

- Peak gain (@1.35 GHz): $4.8 \mathrm{dBi}$

- Output impedance: $50 \Omega$

The coupler is cheap and easy to manufacture since it can be printed on a PCB and does not require machining like other similar sensors. Additionally, its $50 \Omega$ output impedance, curtesy of the infinite balun feed, and low reflection coefficient across the UHF spectrum allow it to interface seamlessly with a plethora of PD detection equipment, from handheld units to

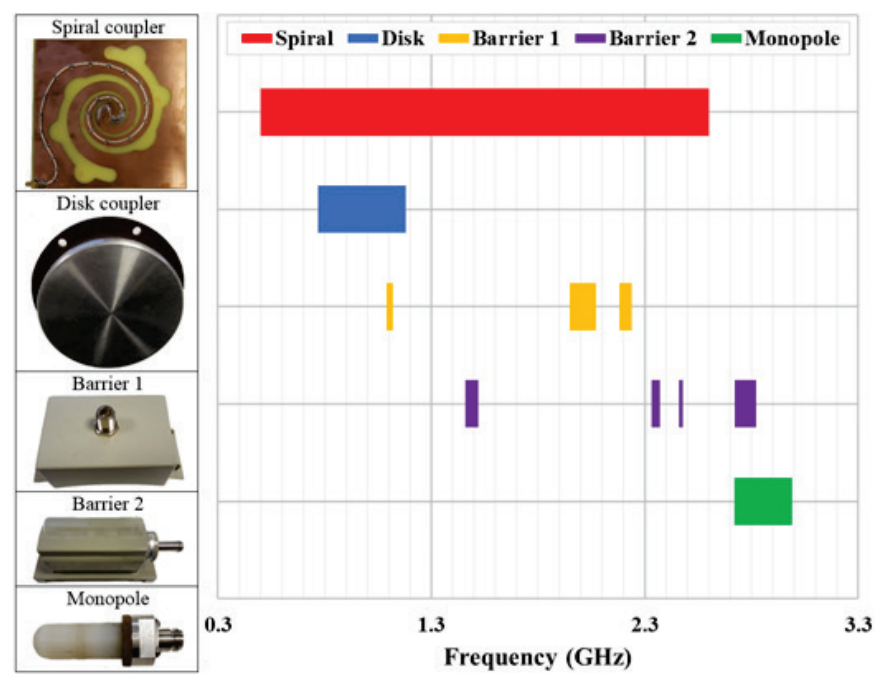

Fig. 16. Comparison of the bandwidth $(\mathrm{S} 11<-9.55 \mathrm{~dB})$ of UHF sensors used for PD detection in GIS. The Spiral UHF coupler has a much wider bandwidth and covers the majority of the UHF spectrum. 
permanent monitors. The coupler's design incorporates a unique feature, the tuning nodules, which extend its bandwidth by more than $35 \%$ without the need for additional components. The wide bandwidth combined with its wide beam width make the spiral UHF coupler an ideal PD sensor for HV equipment such as GIS and transformers since they increase the probability of detecting a variety of defects without the need to use multiple sensors. Furthermore, the coupler can be used to test other UHF PD sensors in a laboratory environment or as a detector of different noise sources on-site, assisting in PD classification.

\section{ACKNOWLEDGMENT}

The work of T. H. Loh was supported by the $2017-2020$ National Measurement System Programme of the UK government's Department for Business, Energy and Industrial Strategy (BEIS), under the Science Theme Reference EMT17.

\section{REFERENCES}

[1] G. C. Stone, "Partial discharge diagnostics and electrical equipment insulation condition assessment," IEEE Transactions on Dielectrics and Electrical Insulation, vol. 12, pp. 891-904, 2005.

[2] The British Standards Institution, "High-voltage test techniques - Partial discharge measurements," BS EN 60270:2001+A1, ed, 2016.

[3] C. Zachariades, R. Shuttleworth, R. Giussani, and R. MacKinlay, "Optimization of a High-Frequency Current Transformer Sensor for Partial Discharge Detection Using Finite-Element Analysis," IEEE Sensors Journal, vol. 16, pp. 7526-7533, 2016.

[4] J. S. Pearson, O. Farish, B. F. Hampton, M. D. Judd, D. Templeton, B. W. Pryor, and I. M. Welch, "Partial discharge diagnostics for gas insulated substations," IEEE Transactions on Dielectrics and Electrical Insulation, vol. 2, pp. 893-905, 1995.

[5] M. D. Judd, Y. Li, and I. B. B. Hunter, "Partial discharge monitoring of power transformers using UHF sensors. Part I: sensors and signal interpretation," IEEE Electrical Insulation Magazine, vol. 21, pp. 5-14, 2005.

[6] J. T. F. 15/33.03.05, "PD detection system for GIS: sensitivity verification for the UHF method and the acoustic method," Électra, pp. 75-87, 1999.

[7] C. Neumann, B. Krampe, R. Feger, K. Feser, M. Knapp, A. Breuer, and V. Rees, "PD measurements on GIS of different designs by nonconventional UHF sensors," CIGRÉ Session 2000, vol. 15-305, 2000.

[8] J. Fuhr, S. Markalous, S. Coenen, M. Haessig, M. Judd, A. Kraetge, M. Krueger, R. Lebreton, E. Lemke, S. Okabe, R. Schwarz, U. Sundermann, S. Tenbohlen, and P. Werle, "Partial Discharges in Transformers," CIGRE, vol. WG D1.29, 2017.

[9] E. W. Weisstein. (2018). Logarithmic Spiral. Available: http://mathworld.wolfram.com/LogarithmicSpiral.html

[10] C. A. Balanis, Antenna Theory: Analysis and Design, $3^{\text {rd }}$ ed. Hoboken, New Jersey: John Wiley \& Sons, 2005.

[11] T. A. Milligan, Modern Antenna Design, $2^{\text {nd }}$ ed. Hoboken, New Jersey: John Wiley \& Sons, Inc., 2005.

[12] H. G. Booker, "Slot aerials and their relation to complementary wire aerials (Babinet's principle)," Electrical Engineers - Part IIIA: Radiolocation, Journal of the Institution of, vol. 93, pp. 620-626, 1946.

[13] J. J. H. Wang and V. K. Tripp, "Design of multioctave spiral-mode microstrip antennas," IEEE Transactions on Antennas and Propagation, vol. 39, pp. 332-335, 1991

[14] Y. W. Zhong, G. M. Yang, J. Y. Mo, and L. R. Zheng, "Compact Circularly Polarized Archimedean Spiral Antenna for Ultrawideband Communication Applications," IEEE Antennas and Wireless Propagation Letters, vol. 16, pp. 129-132, 2017

[15] J. A. Huffman and T. Cencich, "Modal impedances of planar, noncomplementary, N-fold symmetric antenna structures," IEEE Antennas and Propagation Magazine, vol. 47, pp. 110-116, 2005.

[16] J. Dyson, "The equiangular spiral antenna," IRE Transactions on Antennas and Propagation, vol. 7, pp. 181-187, 1959.

[17] W. Frei. (2015). Modeling of Materials in Wave Electromagnetics Problems. Available: http://www.comsol.com/blogs/modeling-ofmaterials-in-wave-electromagnetics-problems/
[18] S. Marburg, "Six Boundary Elements per Wavelength: Is That Enough?," Journal of Computational Acoustics, vol. 10, 2002.

[19] R. Lee and A. C. Cangellaris, "A study of discretization error in the finite element approximation of wave solutions," Antennas and Propagation, IEEE Transactions on, vol. 40, pp. 542-549, 1992.

[20] W. Frei. (2013). Meshing Considerations for Linear Static Problems. Available: http://www.uk.comsol.com/blogs/meshing-considerationslinear-static-problems/

[21] U. R. Kraft, "Polarisation properties of small printed spiral antennas with four resistively loaded arms," IEE Proceedings - Microwaves, Antennas and Propagation, vol. 144, pp. 131-135, 1997.

[22] S. Drabowitch, A. Papiernik, H. D. Griffiths, J. Encinas, and B. L. Smith, Modern Antennas, $2^{\text {nd }}$ ed. Netherlands: Springer, 2005.

[23] T. H. Loh, "Non-invasive measurement of electrically small ultrawideband and smart antennas," 2013 Loughborough Antennas \& Propagation Conference (LAPC), 2013, pp. 456-460.

[24] M. D. Judd, O. Farish, and B. F. Hampton, "The excitation of UHF signals by partial discharges in GIS," IEEE Transactions on Dielectrics and Electrical Insulation, vol. 3, pp. 213-228, 1996.

[25] U. Schichler, W. Koltunowicz, D. Gautschi, A. Girodet, H.Hama, K. Juhre, J. Lopez-Roldan, S. Neuhold, C. Neumann, S. Okabe, J Pearson, R. Pietsch, U. Riechert, and S. Tenbohlen, "UHF Partial Discharge Detection System for GIS: Application Guide for Sensitivity Verification," CIGRÉ, vol. WG D1.25, 2016.

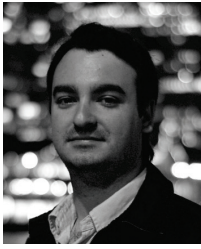

Christos Zachariades was born in Athens, Greece, in 1984. He received the BEng (Hons) in electrical and electronic engineering, in 2009, the MSc in electrical power systems engineering, in 2010, and the $\mathrm{PhD}$ in electrical and electronic engineering, in 2014, from the University of Manchester, UK

He was previously a Senior Test and Development Engineer with HVPD Ltd, UK, and is now a lecturer with the University of Manchester, UK.

Dr. Zachariades is a Chartered Engineer (CEng) and a member of the Institution of Engineering and Technology (IET) as well as a member of the Cyprus Scientific and Technical Chamber (ETEK).

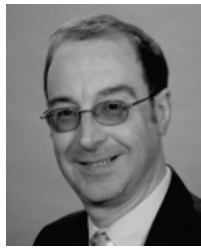

Roger Shuttleworth (M'07) was born in the UK and completed his BSc and PhD degrees in electrical and electronic engineering at The University of Manchester, UK.

He worked for a year at GEC Traction before joining the University as a lecturer in the Power Systems Research group and later the Power Conversion Research group. He has over 100 papers and patents and was Director for the Power Electronics, Machines and Drives MSc course.

His main research activities are in the areas of Power Electronics, Energy Control and Conversion, and Energy Harvesting.

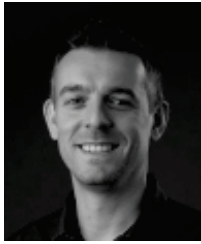

Riccardo Giussani (StM'10, M'13) was born in Legnano, Italy, in 1978. He received the EngD in electrical engineering from Politecnico di Milano, Italy, and the PhD in electrical and electronic engineering from the University of Manchester, UK, in 2015.

Since 2013, he has been working at HVPD Ltd, UK. He is currently HVPD's Principal Development Engineer.

Dr. Giussani is a member of the Institution of Engineering and Technology (IET), the IEEE Dielectrics and Electrical Insulation Society (DEIS) and the IEEE Industry Applications Society (IAS).

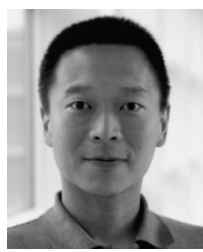

Tian-Hong Loh (S'03, M'05, SM'15) was born in Malaysia and received the BEng from Nottingham Trent University, UK, in 1999, and the PhD from the University of Warwick, UK, in 2005, both in electrical and electronic engineering.

In 2005, he joined the National Physical Laboratory (NPL), UK, where he is currently a Principal Research Scientist. He leads work on applied and computational electromagnetic metrology research. He holds 5 patents and has over 100 publications.

Dr Loh is a Visiting Professor at Surrey University, UK, a Visiting Industrial Fellow at Cambridge University, UK, and an Associate Editor of the Institution of Engineering and Technology (IET) Communications and the International Union of Radio Science (URSI) Radio Science Bulletin for Commission A. 